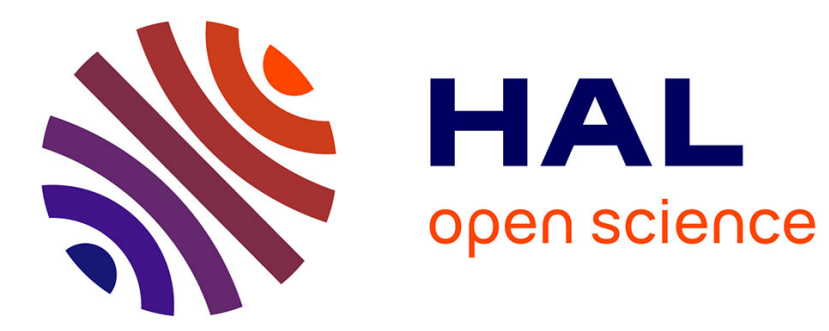

\title{
Professer sa foi en toutes lettres. La correspondance Rousseau-Vernes
}

\author{
Nicolas Brucker
}

\section{To cite this version:}

Nicolas Brucker. Professer sa foi en toutes lettres. La correspondance Rousseau-Vernes. Rousseau en toutes lettres, Mar 2012, Brest, France. pp.207-221. hal-01482995

\section{HAL Id: hal-01482995 \\ https://hal.univ-lorraine.fr/hal-01482995}

Submitted on 13 Mar 2017

HAL is a multi-disciplinary open access archive for the deposit and dissemination of scientific research documents, whether they are published or not. The documents may come from teaching and research institutions in France or abroad, or from public or private research centers.
L'archive ouverte pluridisciplinaire HAL, est destinée au dépôt et à la diffusion de documents scientifiques de niveau recherche, publiés ou non, émanant des établissements d'enseignement et de recherche français ou étrangers, des laboratoires publics ou privés. 


\section{Professer sa foi en toutes lettres : la correspondance Rousseau-Vernes}

Les trente-cinq lettres qui nous sont parvenues de la correspondance Jean-Jacques Rousseau-Jacob Vernes (1754-1765) exposent le processus de mutation d'une amitié si vive qu'elle fait dire au citoyen en 1757: «Je gagne un ami ; je me survivrai dans lui » (lettre 498), aux gémonies auxquelles se vouent les deux hommes après 1762, Rousseau qualifiant alors son correspondant de «méchant sot» (lettre du 15.08.1763). L'intéressant est que nous lisons le processus dans la correspondance elle-même : c'est dans l'échange des lettres, en interaction avec l'œuvre publiée, que s'expriment les divergences de points de vue, menant rapidement à la rupture. Le second intérêt de cette correspondance est qu'elle se prolonge audelà d'elle-même, par une autre forme d'échange, publique celle-là, entre 1763 et 1771, dans le contexte de deux crises : celle ouverte par la Profession de foi du Vicaire savoyard, et qui connaît plusieurs rebondissements, et celle de l'attribution du Sentiment des citoyens ${ }^{1}$. L’homogénéité thématique qui fait le fond de ces échanges est elle aussi remarquable : au point qu'on lit dans cette correspondance une évaluation précise de la dogmatique de Rousseau dans les années concernées. Mais cette lecture nous apprend aussi que la foi de Rousseau est inséparable de son expression, ce qui légitime une approche littéraire de la question religieuse, comprise comme intrinsèquement liée à une poétique de la lettre.

\section{Professer sa foi}

Les lettres les plus connues de cette correspondance sont, à juste titre, les lettres de février (616) et mars 1758 (634), qu'on trouve reproduites dans des choix. S'y expose le sentiment de Rousseau en matière de foi, dans la double actualité de l'article «Genève »- et de la réponse qu'il médite - et de la rédaction de la Profession de foi du Vicaire savoyard, à laquelle il s'attelle. D'une certaine façon, en l'adressant au pasteur Vernes, il essaie cette profession de foi. On peut y lire ceci :

«J'ai de la religion (...). La philosophie n'ayant sur ces matières ni fond, ni rive, manquant d'idées primitives et de principes élémentaires, n'est qu'une mer d'incertitude et de doute, dont le métaphysicien ne se tire jamais. J'ai donc laissé là la raison, et j'ai consulté la nature, c'est-à-dire, le sentiment intérieur qui dirige ma croyance indépendamment de ma raison ». (lettre 616)

\footnotetext{
1 Pour le détail de l'affaire, voir Frédéric S. Eigeldinger, «Des pierres dans mon jardin : les années neuchâteloises de J.-J. Rousseau et la crise de 1765, Paris, Honoré Champion, 1992, p. 207-212.
} 
On croit déjà entendre le Vicaire, dans l'accent et jusque dans les termes employés, réaffirmer la légitimité du sentiment, clair, immédiat, évident, contre les spéculations métaphysiques ${ }^{2}$. A partir de là les dogmes paraissent bien secondaires. «Mon ami, je crois en Dieu, et Dieu ne serait pas juste si mon âme n'était immortelle ». En tant qu'acte libre de la volonté, la foi est première, originelle et originante. A partir d'elle se pensent des contenus, généraux d'abord, l'unité d'intention qui préside à l'existence du créé; dérivés ensuite, la providence ou l'immortalité de l'âme. Rousseau conclut son bref credo par une sentence qui vise à marquer le périmètre strictement subjectif de l'expérience religieuse, périmètre au-delà duquel s'expriment d'autres réalités, non plus spirituelles mais sociales : «Voilà ce me semble tout ce que la religion a d'essentiel et d'utile. Laissons le reste aux disputeurs $»^{3}$. Il poursuit sur la question de la rétribution de la vertu et du vice, et substitue au jugement divin la règle du contentement intérieur. Cela va dans le sens d'une plus grande autonomie morale : l'homme est l'artisan de son bonheur et de son malheur; la sanction vient d'elle-même, selon une justice immanente.

On devine qu'une telle mise au point venait en réponse aux difficultés soulevées par le pasteur sur tel point de doctrine, dans une lettre qui ne nous est pas parvenue. On comprend aussi quelle a pu être la réaction de Vernes à la réception d'une telle confession si peu confessante, d'une confession dont le contenu se résume à la qualité du sentiment, c'est-à-dire du rapport de la conscience à ce qui la fait exister, en l'absence de toute assise théologique contrairement à «l'instinct divin » du piétiste Béat de Muralt, qui est la loi écrite de la main de Dieu dans le cœur de l'homme, un oracle divin, une «parole intérieure » à laquelle chacun doit prêter l'oreille ${ }^{4}$. Cette foi, qui se limite à la sphère du moi, mesure du bien et du mal, de la vertu et du vice, donc de la récompense et du châtiment, d'un moi qui est seul détenteur de son salut, était-elle pour plaire au ministre du saint Evangile à Céligny, membre de la Vénérable Compagnie des pasteurs et de la Société des catéchumènes de Genève ? Un tel credo, qui minore l'énoncé dogmatique, au point de l'abandonner aux vains «disputeurs », qui ne mentionne pas seulement les Ecritures, se rapproche en plus d'un point du piétisme intellectuel de Marie Huber, tel qu'il s'exprime dans les Lettres sur la religion essentielle

\footnotetext{
${ }^{2}$ Après s'être épuisé en vaines recherches, et sur le point de s'abîmer « dans un océan sans rives », le Vicaire a finalement résolu de « resserr[er] [s]a foi dans [s]es notions primitives ». OC IV, p. 624. Quant à Saint-Preux, il affirmera croire « de la religion tout que ce qu['il] en p[eut] comprendre ( Julie, V, 3). OC II, p. 583.

${ }^{3}$ Dans la Lettre à C. de Beaumont, Rousseau précisera ce qu'il entend par «utilité »: «l'essentiel de la religion consiste en pratique », écrit-il alors. $O C$ IV, p. 962. La foi n'est valide que si elle s'actualise dans des actes qui soient conformes aux principes professés. Foi et charité ne sauraient être dissociées.

${ }^{4}$ DE MURALT B. L., L'Instinct divin recommandé aux hommes, s. 1., s. n., 1727. Voir sur la filiation intellectuelle entre Muralt et Rousseau : RADICA G., L'histoire de la raison. Anthropologie, morale et politique chez Rousseau, Paris, Honoré Champion, 2008, p. 344-348.
} 
(1738), que Rousseau connaissait très vraisemblablement ${ }^{5}$. Le rapprochement est d'ailleurs fait dès l'époque, par le sagace Jacob Vernet, dans l'intention évidente de jeter le discrédit sur son adversaire. Comme Marie Huber, Rousseau rejette toute autorité extérieure, et n'accepte de la révélation que ce que Dieu a révélé à tout homme, sans exclusive aucune. Au sujet de l'Evangile, qui n'est qu'un livre «ignoré des trois-quarts du genre humain », nous lisons dans la lettre 634 :

«Non, mon ami, ce n'est point sur quelques feuilles éparses qu'il faut aller chercher la loi de Dieu, mais dans le cœur de l'homme, où sa main daigna l'écrire. O homme, qui que tu sois, rentre en toi-même, apprends à consulter ta conscience et tes facultés naturelles, tu seras juste, bon, vertueux, tu t'inclineras devant ton maître, et tu participeras dans son ciel à un bonheur éternel. »

Suivant le glissement qui substitue l'homme à l'ami, la lettre familière se mue en une instruction magistrale, dans un mouvement d'amplification qui est habituel du genre du sermon, et qu'autorise l'intertexte augustinien ${ }^{6}$. Ce n'est plus l'humilité de la créature confessant sa foi, c'est l'assurance éclatante du prédicateur, telle précisément que le pasteur Vernes la manifeste dans ses prêches avec le succès dont témoignera plus tard son fils ${ }^{7}$. Ce mouvement est celui de la Profession de foi : après l'aveu de ses errements, puis le récit de ses découvertes, le Vicaire exprime avec toute la conviction dont il est capable les conclusions auxquelles il est parvenu. Si Rousseau se projette ici dans son personnage, il place aussi son correspondant dans la position du prosélyte, et cela quatre ans à peine après avoir été réintégré, discrètement il est vrai, dans l'Eglise de Genève. Cette inversion des rôles n'est que piquante en 1758 ; elle deviendra insultante en 1761 quand Vernes, à la lecture de l'Emile, découvrira à quel point il a été joué.

A cette exaspération mutuelle s'ajoute un climat de méfiance, palpable dès cette époque. Chacun sent que l'autre ne lui dit pas tout : à la réserve soupçonneuse de Rousseau sur son activité littéraire correspondent chez Vernes des liens secrets avec des ennemis déclarés du citoyen, notamment Palissot et Fréron, plus tard Tronchin et Voltaire. Irrité par le ton d'inquisition que prend le pasteur sur les matières religieuses, Rousseau le rappelle au ménagement mutuel que se doivent deux amis: «Souvenez-vous surtout que dans cette

\footnotetext{
${ }^{5}$ Yuji Sakakura mentionne dans La pensée pédagogique de Jean-Jacques Rousseau (Tokyo, Kazamashobo, 1998, p. 293, note 1) que la Bibliothèque publique et universitaire de Genève conserve un exemplaire de la $3^{\text {ème }}$ édition du Système des anciens et des modernes... sur l'état des âmes séparées des corps (Amsterdam 1739), acheté par Rousseau en 1754 et annoté en marge.

${ }^{6}$ « Noli foras ire, in te ipsum redi ; in interiore homine habitat veritas ». De vera religione, XXXIX, 72.

${ }^{7}$ VERNES F., Eloge de M. Vernes, Pasteur de l'Eglise de Genève, in Sermons prononcés à Genève par M. le Pasteur Vernes, t. II, Genève, J. F. Didier, 1792, p. 1-35.
} 
dispute, c'est vous qui attaquez mon sentiment, et que je ne fais que le défendre; car d'ailleurs je suis fort éloigné de désapprouver le vôtre, tant que vous ne voudrez contraindre personne à l'embrasser » (lettre 647). Menaçant quand il s'agit de préserver son indépendance spirituelle et morale, il sait cependant solliciter des services. C'est Vernes qui distribue les exemplaires d'hommage de la Lettre à D'Alembert, puis de la Julie, qu'il destine à ses amis genevois.

Ces lettres, importantes sur le fond, en ce qu'elles complètent notre connaissance de la position religieuse de Rousseau, le sont aussi sur la forme. Rédigée dans un style qui souligne la polarisation de la communication et la tension qui en découle, la profession de foi s'accompagne d'un appareil métadiscursif qui en précise la nature. En la désignant par le mot « confidences » (lettre 616), l'auteur signifie clairement que dire sa foi, c'est la dire au présent, dans l'instantané de l'accord de soi avec soi, mais aussi dans la tension de parole qui unit deux consciences, accordées à l'ordre naturel, sincèrement engagées dans la recherche du vrai $^{8}$. C'est donc la qualité de la relation qui garantit la qualité de la profession, et la préserve de toute autorité extérieure, des formules, symboles et autres catéchismes, que celle-ci fait naître. Car la croyance, dès qu'elle prend une forme impersonnelle et interchangeable, dès qu'elle se désolidarise de la conscience et de la parole, se change en préjugé, devient instrument de contrainte et d'oppression.

A l'opposé de la profession de foi selon Rousseau, la prédication, collective et dogmatique, est soupçonnée d'inauthenticité et d'artifice. Bien des années plus tard, Rousseau reprochera à Vernes de l'avoir prêché dans ses lettres, d'avoir adopté un ton de suffisance et de supériorité, d'avoir brandi contre lui la sacrosainte religion. Rien n'illustre mieux cette distance que la réponse au message que Rousseau lui adressa en février 1760, à la suite de la mort de sa première épouse, Marie-Françoise Clarenc.

«La religion seule m'a soutenu, mon cher citoyen; j'ai senti violemment le coup, mais j'ai baisé humblement la main qui l'a porté ; j'ai remercié l'arbitre de nos destinées, de ce qu'il m'a donné pendant une année cette tendre épouse, dont sans doute je n'étais pas digne ; il a retiré son bienfait ; est-ce à moi à me plaindre ? D'ailleurs, bien convaincu que c'est lui qui dirige tous les événements de la vie, puisje douter que dans les coups dont il frappe ses enfants, il n'ait des raisons de sagesse et de bonté ? Faudra-t-il que je me le représente comme un tyran, qui ne nourrit son cœur que du mal qu'il projette, et qui dans sa sombre retraite s'égaye lugubrement en

\footnotetext{
${ }^{8}$ Il importe au Vicaire de trouver une situation appropriée pour le propos qu'il veut tenir au prosélyte. GUENARD F., Rousseau et le travail de la convenance, Paris, Honoré Champion, 2004, p. 75.
} 
voyant couler les larmes des malheureux qu'il a fait? Non, cher Rousseau, mon Dieu, celui que j'adore, est un tendre Père, qui lorsqu'il afflige, fait, comme dit l'Écriture [Is $28,21]$, son œuvre étrangère, et conduit par la main dans le sentier du vrai bonheur des aveugles qui s'en éloigneraient, s'il les abandonnait à eux-mêmes ! (...) Ah, cher Rousseau, que ceux-là sont cruels qui cherchent à ôter à l'homme cette précieuse espérance ! Barbares, qui ne me montrent dans ma mort que l'anéantissement de tout mon être, et dans la mort des personnes qui me sont chères, qu'une poussière froide et éternellement insensible. Quel affreux système, cher Rousseau, qu'il doit bien l'être pour un cœur tel que le vôtre ! (lettre 942)

Adoptant la rhétorique empesée de la piété ecclésiastique, Vernes se pose en chrétien aguerri ou en sage que n'atteignent pas les vicissitudes de la vie. Ce faisant, il prétend montrer à son correspondant que les principes qu'il a défendus dans ses lettres, il les met à présent en application, et qu'il accorde ainsi sa pensée et son action. Le «est-ce à moi à me plaindre » fait écho à la dispute sur la Providence de 1755-56. Vernes entend marquer sa position, en la distinguant de celle que Rousseau manifesta alors : sa soumission est un hommage à la divinité, à son intelligence et à sa sagesse infinie. Il saisit le prétexte de la mort de sa femme, pour en faire un sermon ${ }^{9}$, et ainsi édifier son « cher Rousseau »; et peu après de la littérature, avec les Consolations des misères de ma vie, stances que Rousseau mettra en musique, et qui ne seront publiées qu'en 1781 ; mais aussi pour se faire l'apologiste de la religion contre les esprits-forts. On retrouve bien ici la double fonction, pastorale et apologétique, défensive ou combative, de l'homilétique du XVIII ${ }^{\text {ème }}$ siècle. Il met plus ou moins clairement en question le christianisme de Rousseau, en le priant à demi-mot de choisir son camp, entre philosophes et antiphilosophes. Est-ce là la lettre «fort pédantesque et fort sèche » que Rousseau évoque dans la Déclaration relative à $M$. Vernes, à laquelle il ne daigna pas répondre, ce qui fut, prétend-il, « la source de sa haine contre [lui] » ${ }^{10}$ ? C'est très vraisemblable. Quand il lira Vernes à nouveau, ce sera dans les Lettres sur le christianisme de J.-J. Rousseau.

La correspondance s'achève donc sur un désaccord patent, sur le fond (piétisme rationaliste contre dogmatisme confessionnel) comme sur la forme (style familier contre style pompeux). La profession de foi de Vernes (lettre 942) répond à celle de Rousseau (lettre 616), et fait sentir la distance infranchissable qui sépare l'homme de l'art de l'homme de bien. Cette rupture en annonce d'autres.

\footnotetext{
${ }^{9}$ Vernes est l'auteur d'un sermon Sur le silence dans les afflictions. Sermons, opus. cit., p. 54-69.

${ }^{10}$ Déclaration relative à M. Vernes, in Euvres de J.-J. Rousseau, t. XVI, Paris, Lequien, 1822, p. 15.
} 


\section{Commerce de bonnes gens ou commerce d'outrages?}

Rousseau définit çà et là, dans ses lettres, une poétique de l'épistolaire indissociable d'une éthique sociale, telle qu'il la rêve et la met en pratique dans la Julie. C'est ainsi que les échanges, si décevants, avec le pasteur Vernes, s'accompagnent d'indications qui visent à mettre le destinataire sur la voie d'une pratique épistolaire sensible, accordée à la philia idéale, à la communauté des âmes pures, dans la transparence rêvée qu'il projette à travers ses lettres. Ce commerce est-elle possible ? Rousseau, qui dit avoir faim d'amitié ${ }^{11}$, s'est-il fait illusion sur son compte? Les Confessions nous donnent l'impression d'une extrême lucidité de Jean-Jacques qui du premier coup aurait sondé à la fois l'ambition démesurée et la sécheresse de cœur du pasteur; il n'en est rien. Ou encore Rousseau se serait-il dédoublé : d'un côté la clairvoyance désabusée qui sonde intus et in cute; de l'autre un cœur tout prêt à aimer, qui s'ouvre, attend, espère, encourage même une amitié balbutiante ?

A une question de Vernes au sujet de la Julie, Rousseau répond en le détournant de l'idée que l'ouvrage serait un «roman philosophique»; c'est un «commerce de bonnes gens », lui dit-il (lettre 664). La correspondance selon Rousseau est cet échange confiant entre des amis qui se livrent sans crainte ni méfiance, étant surs d'être lus et entendus pour ce qu'ils sont, en dehors de toute rivalité, calcul ou recherche d'intérêt. La correspondance s'abstrait des logiques sociales et économiques, elle s'abstrait des conflits d'egos, pour mieux manifester l'épanchement des cœurs, l'expression d'une générosité et d'une naturelle abondance, tout au rebours de ce qu'annonce l'épithète «philosophique », où se lit la froideur reptilienne des pures intelligences, qui masque en réalité d'obscures luttes d'intérêt. En proposant à Vernes de venir par lui-même juger de l'ouvrage, à s'en « mêler », à en prendre la « direction »- et le mot a vraisemblablement aussi son sens spirituel -, Rousseau l'invite à rejoindre la société des belles âmes de Clarens, à le rejoindre, lui Jean-Jacques, dans ce pays enchanté où les lettres ne s'écrivent qu'avec le cœur.

Tout imprégné des personnages et des situations dont il peuple Julie, Rousseau littérarise la réalité tout autant qu'il tire des événements de possibles modèles pour ses créations. Il en est ainsi de la première épouse de Vernes et du couple qu'ils forment. Dans une lettre du 6 janvier 1759, il regarde déjà Marie-Françoise Clarenc comme son amie : «Votre épouse quoiqu'étrangère ne le sera point parmi nous. Le mérite et la vertu ne sont étrangers que parmi les méchants » (lettre 760). Il prétend retrouver en elle la mère de famille dont il a fait le portrait dans la Lettre à d'Alembert ${ }^{12}$ : «Voilà la femme qu'il faut honorer »,

\footnotetext{
11 «Ce dont j'ai faim, c'est d'un ami ». (lettre 634)

${ }^{12}$ Lettre à d'Alembert, Paris, Garnier-Flammarion, GF n ${ }^{\circ} 160,1967$, p. 175-176.
} 
écrit-il. Comme un autre Saint-Preux, il se réjouit de cette union: «Je m'attendris en songeant au bonheur de deux époux si bien unis (...). Quand pourrai-je en être témoin ? Quand verserai-je des larmes de joie en embrassant vos chers enfants ? ${ }^{13}$ Un an plus tard, c'est une lettre de consolation qu'il adresse à Vernes. Il compatit au chagrin que celui-ci éprouve de la perte de sa femme, en montrant à quel point sa situation est proche de celle de son ami, pire encore, s'il est possible, car l'objet de son amour vit et lui échappe. « Heureux qui porte encore au fond de son cœur ce qui lui fut cher. Ô croyez-moi, vous ne connaissez pas la manière la plus cruelle de le perdre : c'est d'avoir à le pleurer vivant » (lettre 940). C'est Saint-Preux dans son exil valaisan, séparé de celle qu'il aime ; c'est Jean-Jacques après sa rupture avec Sophie ; tous deux illustrant la citation de Pétrarque qui figure sur la page de titre de l'édition Rey de la Julie. Ce type de transposition tend à créer une complicité entre les correspondants : engagés à poursuivre un même modèle de sociabilité, qui s'abreuve aux sources du romanesque mais aussi de la pensée politique rousseauiste, ils tissent lettre après lettre le réseau du commerce des belles âmes, et mettent ainsi en pratique la théorie de la cité idéale.

Le passage du «commerce de bonnes gens », rêvé, possible, au «commerce d'outrages » (lettre 633, à Sophie d'Houdetot), lui bien réel, se fait à travers une série d'événements qu'il est inutile de retracer. On se reportera à l'article «Vernes » du Dictionnaire de Jean-Jacques Rousseau ${ }^{14}$. La lecture attentive de la correspondance permet cependant d'en mesurer la dramatique dégradation étape par étape. Y apparaît très nettement le rôle joué en sous-main par Théodore Tronchin et Charles Bonnet, qui ont poussé Vernes à publier ses Lettres sur le christianisme de J.-J. Rousseau, y ont peut-être collaboré, comme le suggère R. A. Leigh, en tout cas ont cherché à distendre et à rompre la liaison VernesRousseau, afin de mieux isoler le citoyen. De son côté, Vernes cherche après l'interdiction de son catéchisme par la Vénérable Compagnie, à donner des gages à l'Eglise de Genève, et à assurer grâce à cette polémique sa publicité à la face de toute l'Europe, Rousseau lui servant de faire-valoir. Ses Lettres signifient la fin de la correspondance privée et ouvrent la période des querelles publiques. La relation, désormais médiée par l'opinion, engage non seulement deux individus, mais deux auteurs, et les missions dont chacun se sent investi au regard de la société. C'est le rôle d'apologiste que Vernes endosse naturellement.

\footnotetext{
${ }^{13}$ Voir Julie, II, 16, OC II, p. 244.

${ }^{14}$ CANDAUX J.-D., «Vernes », R. TROUSSON et F. S. EIGELDINGER (dir.), Dictionnaire de Jean-Jacques Rousseau, Paris, Honoré Champion, 2006, p. 915-916.
} 
La première lettre (2018, du 21.7.1762) adressée par Vernes à Rousseau après un silence de deux ans et demi, a pour but de justifier l'auteur des Lettres sur le christianisme de J.-J. Rousseau ; elle est éclairante de la conception qu'il se fait de la profession de foi.

«Quand tout ce que vous avez dit sur le christianisme serait fondé, quel bien feriezvous à la société en lui enlevant un de ses plus fermes appuis ? Quelles angoisses vous avez mises dans de bonnes âmes, en voyant des doutes proposés, avec toute la force possible par un homme dont on adore les talents et le génie !»

Autrement dit, le doute intérieur doit rester dans la sphère de la communication privée, il ne peut s'exprimer publiquement, même dans les formes d'énonciation sophistiquée qui en préviendraient toute identification à l'auteur, comme c'est le cas dans la Profession de foi. Rousseau, avec ce que Vernes appelle ses «difficultés sur le christianisme », s'est incidemment rangé au côté des philosophes. Il a «troublé les âmes affermies dans la foi, enlevé ce qui en restait dans quelques autres, et fait triompher [les] libertins, qui s'appuient de l'autorité d'un homme tel que [lui]». Sans être intentionnellement un détracteur de la religion, Rousseau joue le jeu des ennemis du christianisme; thème qu'on retrouve plus tard dans les romans antiphilosophiques, et que Vernes va lui-même exploiter.

Transférée à la sphère publique, la correspondance est divulguée et analysée. Chacun des deux écrivains se livrent à cet exercice, Vernes en publiant les lettres échangées en 1765 relativement à l'affaire du Sentiment des citoyens, en guise d'apologie ; Rousseau, en projetant de publier les mêmes lettres, et en y joignant un petit mémoire justificatif : c'est la Déclaration relative à M. le Pasteur Vernes, qui en fait ne sera publiée par DuPeyrou qu'en 1790. A la correspondance se superpose une méta-correspondance en forme d'auto-édition critique, analysant et interrogeant l'ensemble des lettres échangées, et jusqu'à la nature du lien qui unissait les deux hommes, le but étant de déterminer ce qui est imputable à l'un ou à l'autre. «Imputer» : le verbe est au cœur de la polémique. Vernes se plaint à Rousseau de ce qu'il lui a imputé le Sentiment des citoyens, tandis que Rousseau reproche à Vernes de lui avoir imputé la Profession de foi du vicaire savoyard sans égard aux précautions énonciatives qu'il a eu soin de prendre.

\section{L'orthodoxie en question}

Si Vernes intente à Rousseau un procès en hétérodoxie, dont il instruit la cause à partir de 1762 dans une série d'ouvrages de nature polémique, le philosophe et son entourage ne manquent pas de répliquer, parfois en utilisant les mêmes armes. Ainsi de la Déclaration dans laquelle Rousseau présente son adversaire comme « un prédicateur, qui jusque-là n'avait point 
été dévoré du zèle de l'orthodoxie ${ }^{15}$, allusion transparente au Catéchisme de 1761, que Vernes avait présenté à la Vénérable Compagnie, sans succès, puisque refusé, il fut saisi et détruit, au point qu'il n'en reste aujourd'hui plus aucune trace. Ce catéchisme, destiné à remplacer la version déjà ancienne d'Ostervald, fut accusé de libéralisme et jugé sur plus d'un point contraire à la bonne doctrine. L'idée de ressortir cette affaire fut suggérée par Moultou, lui qui dans une lettre à Usteri qualifiait ce même ouvrage de «catéchisme socinien » (lettre $2995)$; ce qui est une manière de répliquer aux anathèmes lancés par Tronchin, qui voit dans la Profession de foi un «code complet de déisme » (lettre 1867).

Plus nuancée est l'analyse de Moultou dans une lettre qui tente d'infléchir la position de Vernes. A la question que celui-ci lui adresse de savoir s'il faut « être absolument chrétien comme Rousseau » (lettre 2800 de juillet 1763), il répond en le renvoyant à ses propres variations : «Le christianisme de votre catéchisme n'était pas celui de M. Ostervald, M. le Professeur Pictet n'était pas chrétien comme M. Turretin, ni M. Maurice le père comme M. Vernet». En citant les noms de théologiens genevois, Moultou rappelle que la doctrine a subi un sérieux infléchissement depuis la Formula consensus de 1675, que l'orthodoxie éclairée de Benedict Pictet, héritage de son oncle François Turretin, a peu à voir avec le libéralisme de son fils Jean-Alphonse, qui marque précisément la transition entre orthodoxie et Lumières, qu'enfin Genève est devenue dès 1728, selon le témoignage d'Ostervald, le haut-lieu de l'hétérodoxie, la tolérance religieuse allant jusqu'à admettre la profession publique d'idées arminiennes, voire sociniennes ${ }^{16}$. L'avenir donnera d'ailleurs raison à Moultou. En 1776, Vernes fait paraître un nouveau Catéchisme, qui cette fois sera agréé par la Compagnie, même si celle-ci ne l'adoptera pas officiellement. Ni pleinement rationaliste, ni pleinement orthodoxe, il offre un visage composite, reflet de la position de l'Eglise genevoise. Il escamote les articles dogmatiques les plus sensibles, tels que les miracles, l'autorité des Ecritures, la rédemption, les mystères, préférant développer la preuve physico-théologique ${ }^{17}$. Voltaire avait vu juste dans sa raillerie : en le rangeant au nombre des sectateurs du «pur déisme », de cette religion «simple et raisonnable » professée par les anciens brachmanes, il avait démasqué celui qu'il nommait affectueusement son «cher prêtre ». Mais il avait aussi jugé son homme, et évalué sa lâcheté à manifester hautement ses convictions ${ }^{18}$.

\footnotetext{
${ }^{15}$ Opus cit., p. 16.

${ }^{16}$ PITASSI M.-C., De l'orthodoxie aux Lumières. Genève 1670-1737, Genève, Labor et Fides, 1992.

${ }^{17}$ PITASSI M.-C., « Le catéchisme de Jacob Vernes ou comment enseigner aux fidèles un "christianisme sage et raisonnable" », Dix-huitième Siècle, n 34, Paris, PUF, 2002, p. 213-223.

18 «Vous seriez bien étonné de trouver dans ce manuscrit [le pseudo Ezour-Veidam] quelques-unes de vos opinions ; mais vous verriez que les anciens brachmanes, qui pensaient comme vous et vos amis, avaient plus de courage que vous ». Lettre à Vernes du 1.10.1761.
} 
Vues d'un peu loin, les convergences entre Vernes et Rousseau seraient plus frappantes que les divergences. A quelques années de là, dans le camp catholique, l'abbé Boulogne portera sur les deux acteurs de la querelle un jugement égalisateur qui les renverra dos à dos. Le socinianisme de Rousseau rejoint sous la plume du journaliste le socinianisme de Vernes ${ }^{19}$. Le reportage de d'Alembert sur la situation religieuse à Genève se trouve validé quarante ans plus tard, et les deux adversaires enveloppés dans une commune condamnation. La différence entre les deux hommes tiendrait dans la manière d'ordonner la communication de la profession de foi. Proposer ses doutes semble à Vernes contre-productif. Et d'abord parce que ce n'est pas ainsi qu'on fait carrière. Lui-même, tiraillé entre la perspective d'être un Fréron protestant, apologiste inflexible de la religion maltraitée, et celle d'être un théologien libéral, ami des philosophes et connu de l'Europe lettrée, ne sut quelle option prendre. C'est bien l'image d'un homme indécis qu'il laissera à la postérité.

Qui des deux hommes avait le premier commencé à jouer le jeu dangereux de l'exclusion? En 1759, au sommet de son prestige, Rousseau se pose en défenseur de la République helvétique, menacée par le mauvais air de Paris, et aussi par le «maître en plaisanteries » qui a établi ses quartiers non loin. Pervertis, les Genevois seraient devenus étrangers à leur patrie: Genève n'est plus dans Genève (lettre 833). Trois ans plus tôt, il sommait déjà Vernes de choisir son camp. Il cherchait à détourner l'auteur du Choix littéraire, sorte de nouveau Mercure Suisse, médiocre compilation de pièces françaises et étrangères, des frivolités littéraires, pour l'engager à se saisir de matières plus gravement utiles, comme cette Histoire de Genève, entreprise en collaboration avec Roustan (lettre 286). Il l'incite à prendre la plume contre les ennemis de la République : "Cher Vernes, soyons hommes et citoyens jusqu'au dernier soupir. Osons parler pour le bien de tous, fût-il préjudiciable à nos amis et à nous-mêmes » (lettre $664 \mathrm{du}$ 4.7.1758). C'est précisément ce que Vernes ne saurait entendre. Sa philosophie ne s'élève pas au point de lui faire oublier son intérêt personnel. Il retournera cependant l'accusation contre Rousseau, qu'il présente dans les Lettres comme un être louvoyant entre matérialisme athée et spiritualisme évangélique, d'autant plus dangereux qu'il n'est pas un ennemi déclaré ${ }^{20}$.

Rousseau trouble les pasteurs de Genève, dans la mesure où il leur renvoie l'image de leur religion telle qu'elle se destine à être, telle que la décrit d'Alembert, « un socinianisme

\footnotetext{
${ }^{19}$ BOUlOGNE E. A., Euvres de M. de Boulogne. Mélanges, t. III, Paris, Le Clère, 1828, p. 404-415. L'article, paru en 1802, traite du Catéchisme à l'usage des jeunes gens de toutes les communions chrétiennes de Vernes (Londres, 1785).

${ }^{20}$ «Un ennemi n'est jamais plus à craindre que quand on le croit ami ; ses coups n'en sont que plus assurés ». Lettres sur le christianisme de J.-J. Rousseau, Genève, E. Blanc, 1763, p. 105-106.
} 
parfait ». A ce titre il représente un double honni, la part inavouable d'eux-mêmes. La correspondance Rousseau-Vernes permet d'affiner l'analyse. Au cours des échanges de lettres se mettent en place les éléments qui composent l'image totale de l'autre en soi, en lien avec un arrière-plan littéraire : l'amitié (roman pastoral), l'héroïsme républicain (registre épique), l'héroïsme chrétien ou martyre (histoire sainte). Rousseau est rêvé par Vernes, un Rousseau fréronisé, polémiste infatigable, ardent défenseur d'une orthodoxie dont on a vu à quel point elle était chimérique. Vernes quant à lui est rêvé par Rousseau en républicain fidèle aux vertus ancestrales, en sage chrétien, un nouvel Abauzit en somme, mais aussi en tendre époux et en ami inséparable. Ce faisant, chacun se peint tel qu'il se rêve à travers l'autre. L'écriture de la lettre peint moins le destinataire que le scripteur ; ce miroir d'encre nous fait voir une image insolite de Rousseau, en pasteur libéral de l'Eglise de Genève. On s'explique mieux dans ces conditions l'énergie qu'il emploiera, une fois que le mirage se sera dissipé, à vouloir effacer toute trace de cette liaison épistolaire, la ténacité qu'il mettra à déconstruire pièce par pièce la chimère qu'il s'était créée. Cette entreprise est menée avec d'autant plus de détermination que Vernes poursuit plus obstinément son plan d'annexion de la pensée et de l'œuvre de Rousseau à sa cause. Les Lettres tendent à imposer du philosophe l'image d'un chrétien dévoyé, dont «l'étrange religion » est au rebours de tout christianisme ${ }^{21}$. Il lui oppose le destin qui aurait été le sien s'il avait « consacré son pinceau de feu à la défense du christianisme, au lieu d'en forger une arme pour le combattre $»^{22}$. Le portrait d'un Rousseau à l'irréel du passé permet de faire surgir la figure contrapunctique d'un anti-Vicaire, sorte de Vernes fantasmé, professant une foi normalisée. Mais c'est à la fiction que Vernes réserve, en 1771, l'invention du personnage d'un Rousseau ennemi déclaré du christianisme, auteur d'un symbole susceptible d'inspirer les sectateurs du philosophisme réunis en synode. La Confidence philosophique (1771) noircit encore Rousseau, tout en lui rendant hommage. Les emprunts à l'œuvre du citoyen, à commencer par la Profession de foi, sont légion. Il n'en reste pas moins que ce roman n'est guère qu'une capucinade. Le mot, employé par Rousseau en réponse à un commentaire de Vernes sur le dénouement de Julie (lettre 1436 du 24.6.1761), est d'ailleurs repris au début de ce petit roman par lettres, pour qualifier le propos moralisateur tenu par Darington dans la lettre qu'il a adressée au jeune débauché Torman dans l'intention de le convertir $^{23}$. La Confidence philosophique est pleine de ces échos, à la cohérence souvent

\footnotetext{
${ }^{21}$ Lettres, op. cit., p. 101. L'Examen de ce qui concerne le christianisme (...) de J.-J. Rousseau (1765) poursuit la charge en contestant à Rousseau le titre de chrétien : sa profession de foi est contraire au christianisme, elle rejette le catholicisme tout autant que le protestantisme.

${ }^{22}$ Lettres, op. cit., p. 103.

${ }^{23}$ Confidence philosophique, Londres [Genève], 1771, p. 281.
} 
problématique si l'on garde à l'esprit la nature polémique de l'ouvrage, mais qui traduisent une indiscutable fascination pour l'œuvre de Rousseau.

De son côté, Jean-Jacques, à partir du point terminal que représente l'affaire du Sentiment des citoyens, se met à réécrire l'histoire de sa relation avec Vernes. Il joint à l'édition des lettres échangées en 1765 une apologie dont deux pages du livre XII des Confessions rendent une synthèse ${ }^{24}$. La diabolisation de Vernes y est complète. Alors que Rousseau tente de réduire au néant d'un événement qui n'aurait pas eu lieu la liaison épistolaire qu'il eut avec le pasteur, Vernes cherche au contraire à prolonger le commerce brutalement interrompu dans des dialogues fictifs : entretiens entre un chrétien et un lettré chinois $^{25}$, dialogue entre Eraste et Eusèbe ${ }^{26}$, échanges verbaux et épistolaires entre Torman et Darington $^{27}$, parmi bien d'autres dialogues secondaires et sans compter les apostrophes rhétoriques à Rousseau ${ }^{28}$. Si caricaturaux qu'ils soient, ces doubles des deux correspondants poursuivent, sous la plume de Vernes et sous sa direction, l'entretien jadis commencé. Mais Vernes, dans ses trois ouvrages apologétiques, ne se contente pas de transposer en la simplifiant la dispute engagée au temps de Montmorency. L'apologiste, qui avec méthode réfute une thèse, se double d'un lecteur qui, passionnément, discute du sens des textes qu'il admire, et qui, en se confrontant à la figure, mythifiée dès 1760, de leur auteur, en partage tant soit peu le prestige. Avec ou contre Rousseau, c'est toujours à travers le philosophe-citoyen que Vernes accomplit son propre destin littéraire.

La relation Rousseau-Vernes s'ordonne toute autour de la question de la profession de foi. Aussi la lettre 616 apparaît-elle comme un point de départ, mais sans doute également comme un point d'arrivée. Car si éloigné que Vernes ait pu paraître de Jean-Jacques, au point de se donner pour mission la réfutation ou la correction de ce qu'il juge être ses errances confessionnelles, il demeure dans la périphérie immédiate des articles de foi rousseauistes, et dans la dépendance des prises de position successives du philosophe. A partir de 1758, Vernes ne cesse de discuter ce dernier, sur l'esprit comme sur la lettre, mettant sa réflexion en orbite autour de la planète Rousseau; chaque déclaration du philosophe en matière de religion suscite chez le pasteur une réponse, chaque mouvement de l'un engendre chez l'autre un

\footnotetext{
${ }^{24}$ OC I, p. 632-634.

${ }^{25}$ Lettres, op. cit., p. 91-102.

${ }^{26}$ Examen de ce qui concerne le christianisme (...) de J.-J. Rousseau. Eraste est un disciple de Rousseau, tandis que Vernes est un porte-parole de Vernes.

${ }^{27}$ Notamment la longue lettre de Darington qui commence par une confession («A l'âge de vingt ans, je me fis, comme vous, incrédule (...) ») et se poursuit par une réfutation systématique des éléments de la profession de foi rousseauiste. Confidence philosophique, op. cit., p. 237-281.

${ }^{28}$ «Qu'il me soit permis d'adresser ici la parole à l'auteur d'Emile ». Lettres, op. cit., p. 69.
} 
mouvement analogue. Même si son raisonnement est le plus souvent biaisé et sans vraie pertinence, Vernes n'en est pas moins le prosélyte d'un Vicaire qui ne se serait pas bien fait comprendre, ou dont tout le pouvoir de persuasion ne pourrait venir à bout des résistances que l'autre lui opposerait. Ou bien encore Rousseau serait-il la mauvaise conscience du pasteur? La profession de foi par laquelle son porte-parole conclut les Lettres sur le christianisme de J.-J. Rousseau reprend, pour l'écarter, la formule des «chrétiens en effigie », tirée de la Lettre à C. de Beaumont ${ }^{29}$. Se sentant visé par cette accusation, il cherche à s'en défendre en se déclarant chrétien par la pratique et par la croyance, par la foi et par les œuvres. Il est en réalité tiraillé entre une discipline évangélique d'inspiration paulinienne et l'imprescriptible autonomie de la conscience croyante prônée par Rousseau. La critique rousseauiste du dogmatisme l'engage à s'interroger à son tour, et à redéfinir le fondement de son christianisme, à partir de quoi il imaginera un symbole commun à tous les chrétiens. Après la notion d'orthodoxie, il met en question le genre du catéchisme dans son contenu comme dans sa lettre. Cet infatigable censeur de Rousseau cherche dans la controverse la voie d'un possible renouvellement de la profession de foi réformée, tant sur le fond que sur la forme.

Mais plus que tout, ce qui oppose les deux hommes c'est une conception différente du statut de la parole confessante. Chez Rousseau, G. Radica le note avec raison, est affirmée l'unité d'un rapport discursif à l'être. Celui-ci ne peut être atteint autrement que par le discours, même inexact, qui le donne. C'est pourquoi «les croyances sont indissociables de certains discours propres à les entretenir et à les faire naître $»^{30}$. Vernes, au contraire, tient que la vérité existe indépendamment de la parole qui l'exprime. De là le malentendu qui, dès l'origine de la correspondance, a creusé la distance entre les deux hommes. De là l'irritation de Rousseau à ne pas être compris, ou tout simplement lu. La lecture, comme l'écoute, suppose un décentrement de soi qui puisse faire place à ce qui advient, en dehors des cadres de la norme, de l'habitude ou du prévisible. Lire une profession de foi, ce sera permettre à cette vérité d'émerger, malgré ou grâce à l'approximation des mots - et Rousseau revendique une telle approximation -, dans sa singularité et son unicité. Cette vérité n'est ni extérieure ni indifférente; elle est ce qui fait vivre, puisqu'elle justifie l'individu dans son rapport à l'existence. Face à cet enjeu, que pèsent les subtiles disputes théologiques ? Que Rousseau veuille se livrer sans réserve, en recourant à toutes les ressources sensibles du langage, dans une confidence qui est aussi un témoignage de confiance, n'a donc rien qui surprenne. Qu'il

\footnotetext{
${ }^{29}$ Lettres, op. cit., p. 128. Lettre à C. de Beaumont, op. cit., p. 962.

${ }^{30}$ RADICA G., op. cit., p. 666.
} 
s'indigne des querelles qu'on lui cherche, qu'il choisisse de se retrancher dans un silence hostile dès qu'il s'en sent la cible, n'est pas davantage étonnant.

Ce n'est pas seulement d'un ami dont Rousseau avait faim, mais d'un lecteur attentif, à l'écoute empathique et bienveillante. Ne le pouvant trouver dans le réseau de ses correspondants, il choisit de l'imaginer, dans des personnages fictifs ou dans l'anonyme opinion. Parallèlement au régime confidentiel de sa profession, il élabore, sur un mode identique, une expression publique de son sentiment religieux. L'attitude attendue du lecteur de ces confessions de foi n'est pas différente de celle que l'auteur requiert de ses correspondants. La correspondance privée modélise ainsi l'œuvre publique ; l'une et l'autre obéissent aux mêmes règles poétiques et nourrissent un rêve unique, d'être enfin lu, entendu, reconnu. Ce rêve s'éloigne à mesure que s'amplifie le scandale, que se multiplient réfutations, pamphlets et libelles, et qu'il n'est plus d'ami pour recevoir en toutes lettres la profession de foi d'un cœur sensible.

Nicolas Brucker

Université de Lorraine

Centre Ecritures (EA 3943)

Metz, F-57000, France 